\title{
The retina dose-area histogram: a metric for quantitatively comparing rival eye plaque treatment options
}

\author{
Prof. Melvin A. Astrahan \\ Department of Radiation Oncology, University of Southern California School of Medicine, Los Angeles and Eye Physics, LLC, Los Alamitos, \\ California, USA
}

\begin{abstract}
Purpose: Episcleral plaques have a history of over a half century in the delivery of radiation therapy to intraocular tumors such as choroidal melanoma. Although the tumor control rate is high, vision-impairing complications subsequent to treatment remain an issue. Notable, late complications are radiation retinopathy and maculopathy. The obvious way to reduce the risk of radiation damage to the retina is to conform the prescribed isodose surface to the tumor base and to reduce the dose delivered to the surrounding healthy retina, especially the macula. Using a fusion of fundus photography, ultrasound and CT images, tumor size, shape and location within the eye can be accurately simulated as part of the radiation planning process. In this work an adaptation of the dose-volume histogram (DVH), the retina dose-area histogram $(\mathrm{RDAH})$ is introduced as a metric to help compare rival plaque designs and conformal treatment planning options with the goal of reducing radiation retinopathy.

Material and methods: The RDAH is calculated by transforming a digitized fundus-photo collage of the tumor into a rasterized polar map of the retinal surface known as a retinal diagram (RD). The perimeter of the tumor base is digitized on the RD and its area computed. Area and radiation dose are calculated for every pixel in the RD.

Results: The areal resolution of the RDAH is a function of the pixel resolution of the raster image used to display the RD and the number of polygon edges used to digitize the perimeter of the tumor base. A practical demonstration is presented.

Conclusions: The RDAH provides a quantitative metric by which episcleral plaque treatment plan options may be evaluated and compared in order to confirm adequate dosimetric coverage of the tumor and margin, and to help minimize dose to the macula and retina.
\end{abstract}

Key words: eye plaque, episcleral plaque, brachytherapy, retina, dose-area histogram.

\section{Purpose}

The documented use of brachytherapy in the treatment of choroidal melanoma dates back to the use of radon seeds in the late 1930s, and episcleral plaques embedded with the radionuclide Co-60 were described by Stallard [1] in the 1960s. Another plaque of that era, which remains in use to this day, employed the embedded radionuclide Ru-106 [2,3]. In subsequent years plaques containing I-125 [4] and Ir-192 [5] seeds were introduced. A significant advance in eye plaque technology occurred in the mid 1980s, when the Collaborative Ocular Melanoma Study (COMS) introduced a set of standardized plaques designed for I-125 seeds [6]. The selection of the low energy I-125 radionuclide by the COMS, and more recently Pd-103 by others [7], meant that a plaque with a thin gold shell would be sufficient to block essentially all of the primary radiation that would otherwise irradiate the patient's brain and orbit as well as the surgeon and other attending personnel.

As illustrated in Fig. 1, intraocular tumors such as choroidal melanoma typically appear as small dome-shaped mounds rising inward from the scleral shell of the eye. The plaque containing the radionuclide is surgically positioned on the sclera below the base of the tumor and then sutured to the sclera to affix the plaque at that location for the 3 to 7 day duration of the implant.

The most notable vision-impairing complications subsequent to episcleral plaque therapy are cataract, radiation maculopathy $(\mathrm{RM})$ and to a lesser extent radiation retinopathy (RR), optic neuropathy and neovascular glaucoma [8]. Cataracts are now of lesser concern owing to the introduction of intraocular lens (IOL) replacements. It was recently reported [9] that an antibody to vascular endothelial growth factor (VEGF) which inhibits neovascularization and reduces 


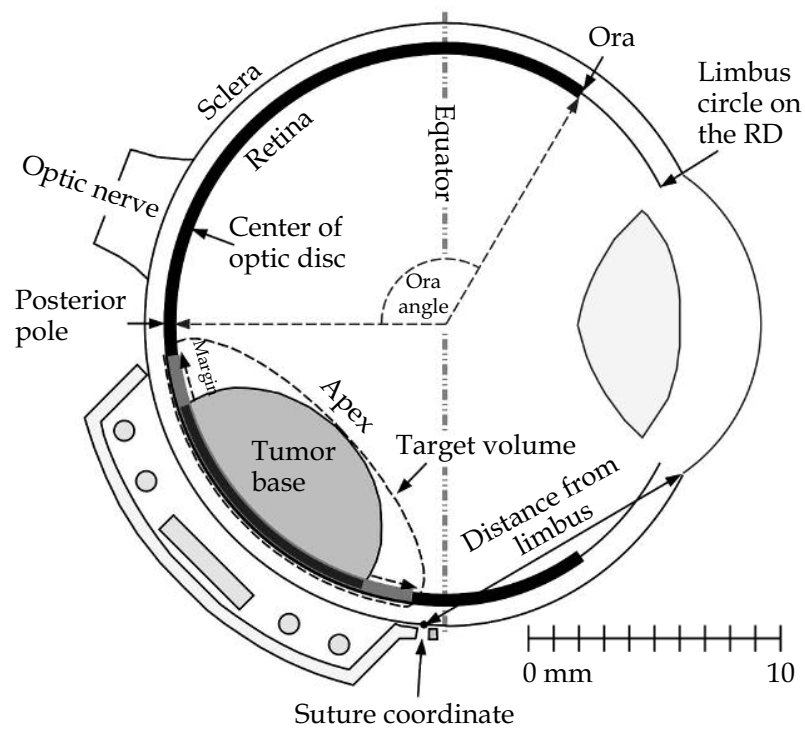

Fig. 1. A $5 \mathrm{~mm}$ tall, $10 \mathrm{~mm}$ diameter posterior tumor with a $2 \mathrm{~mm}$ retinal margin surrounding the base of the tumor. Posterior to the equator the sclera is modeled as a hemisphere. Anterior to the equator, it is modeled as an oblate spheroid which intersects the cornea at the limbus. The photosensitive portion of the retina, drawn as the thick black line, is modeled as being inset $1 \mathrm{~mm}$ from the exterior of the sclera and extending from the posterior pole to the ora serata

retinal edema appears to reduce the severity of RM. These complications are progressive and may have a delayed onset of months to years after radiation treatment. The severity is dependent on the dose rate, the total dose of radiation and the amount of retina irradiated.

The obvious way to reduce vision-impairing complications is, to the extent practical, to conform the prescribed isodose surface to the target volume and reduce the dose delivered to those photosensitive portions of the retina that fall outside of the target volume, with particular attention to the macula. The dose prescription target volume for intraocular tumors, illustrated in Fig. 1, is typically defined by the apex of the tumor and a small retinal margin (e.g. $2 \mathrm{~mm}$ ) surrounding the tumor base. This margin accounts for microscopic extension of the tumor and uncertainties associated with plaque placement. This margin is analogous to the planning target volume (PTV) concept. Therefore, the objective of conformal plaque therapy is to enclose the tumor apex and base + margin within the prescribed isodose surface while sparing as much of the surrounding tissue as possible.

Fortunately, the gold shell of the plaque absorbs virtually all primary radiation emanating from the radionuclide seeds that is initially directed away from the eye, and because the dome portion of the tumor is surrounded by radiobiologically inert vitreous humor, it is not critical for the isodose surface to closely conform to the shape of the dome in order to achieve the radiobiological goals of conformal therapy. It is primarily the dose delivered to the target volume and to the retina that are of radiobiological concern, and therefore it is sufficient that the prescribed isodose surface covers, and preferably conforms to, the shape of the base (+ margin) of the tumor.

Image-based treatment simulation software for ophthalmic plaques that could model the conformal treatment of intraocular tumors was initially developed and adopted for clinical use at the University of Southern California (USC) in the early 1990s and has been in continuous development ever since [10-16]. This software fuses fundus photography, ultrasound, and either computed tomography (CT) or magnetic resonance (MR) images to build interactive three-dimensional (3D) virtual models of each patient's ocular anatomy, the plaques and the radiation sources. The software was licensed for commercial distribution in 1994 under the title Plaque Simulator ${ }^{\mathrm{TM}}$ (Eckert \& Ziegler BEBIG GmbH, Robert-Rössle-Str.10, D-13125 Berlin, Germany). Gold plaques featuring collimating slots for each radiation source, introduced in the late 1990s [15] demonstrated that highly conformal treatment of ocular tumors was possible.

A fundamental requirement of all conformal external beam radiotherapy treatments (e.g. radiosurgery) is precise patient and beam positioning. A conformal isodose plan with a steep dose gradient outside the target volume is of little clinical value if it cannot be reliably delivered. An analogous situation applies to conformal plaque treatment. If the plaque is not positioned on the eye at the location and orientation intended by the plan, then portions of the tumor may receive less dose than intended, and the healthy retina could receive more dose than intended.

Historically, the information regarding tumor size, shape and location within the eye that was often provided for plaque treatment planning purposes consisted of approximations and hand-drawn sketches. Occasionally, at the time of surgery, a plaque might be found to be too small, perhaps due to intervening tumor growth, requiring the plaque to be reconfigured while the patient remained under sedation or risk inadequate coverage of the tumor base. The position on the eye at which the plaque would be affixed was not determined until the time of surgery during which a bright light was used to transilluminate the eye and cast a shadow of the tumor base onto the underlying translucent sclera. This shadow was traced with a marker pen and the plaque positioned to cover the tumor base. In aviation parlance, this approach is analogous to "flying by the seat of one's pants" compared to using a predetermined flight plan and instruments. Under these conditions, conformal treatment and calculation of dose to the retina was not practical.

Using image-based 3D treatment simulation, the tumor location can be accurately determined and plaque position can be planned in advance of surgery. To facilitate accurate positioning at the planned position, the simulation software provides targeting support in the form of suture eyelet coordinates on the sclera in a format familiar to the ophthalmic surgeon. Coordinates are expressed as a meridian clock hour (see Fig. 3) and a chord distance from the limbus (see Fig. 1) for each suture eyelet of the plaque [12,16]. In practice, the meridian clock hours can be estimated to with a few minutes by the surgeon, and the chord distance on the meridian 
precisely measured using an adjustable Castroviejo caliper. The $2 \mathrm{~mm}$ PTV margin surrounding the tumor base takes into account the uncertainty in locating the meridian. Nearly two decades of experience using this coordinate system at USC has demonstrated that it is simple and reliable. Providing suture coordinates also frees the plaque shell from having to physically conform to the shape of the tumor base, it is sufficient that the radiation sources mounted within the shell provide the conformal isodose coverage when the shell is positioned as planned. Freeing the shape of the shell from the physical shape of the tumor enables the suture eyelets to be relocated to more convenient anterior locations where suturing is easier and more accurate.

Although both the hardware, software and a targeting system now exist for planning and delivering conformal treatment of intraocular tumors, until now a means of quantitatively comparing rival treatment plan options has been lacking. In conventional radiation therapy treatment planning, the dose-volume histogram [17] (DVH) has proven itself a useful tool for comparing rival treatment plans. In this work an adaptation of the DVH concept, the retina dosearea histogram (RDAH) is introduced as a quantitative tool for the comparison of rival plaque designs and treatment plan options with the ultimate goal of reducing radiation retinopathy.

\section{Material and methods}

The Plaque Simulator ${ }^{\mathrm{TM}}$ (PS) software [10-16] was modified to version 5.7 to support the simultaneous calculation and display of RDAHs for up to 4 rival plaque configurations. In this version of the software histograms are calculated for the photosensitive region of the retina between the posterior pole and the ora serrata, the macula, the tumor base, the base plus retinal margin and for photosensitive retina outside the base and margin. In the PS software, the sclera posterior to the equator is modeled as a $1 \mathrm{~mm}$ thick hemispherical shell. Sclera anterior to the equator is modeled as a $1 \mathrm{~mm}$ thick oblate spheroidal shell which intersects the dome-shaped cornea. The $1 \mathrm{~mm}$ scleral shell thickness is an assumption carried forward from the model of the eye that was recommended by the COMS, but it appears to be adequate for treatment planning purposes.

Referring again to Fig. 1, the photosensitive portion of the retina is modeled as a lining covering most of the interior surface of the sclera. The ora serrata delineates the transition from the simple non-photosensitive area of the retina near the ciliary body to the complex, multi-layered photosensitive region. The location of the ora serrata in the computer model is specified by a user adjustable ora-angle. Describing the photosensitive extent of the retina by a single angle enables the model to adapt to eyes of different spherical and oblate radii of curvature with minimal user intervention. The PS default for this angle is 123.3 degrees.

The determination of the size, shape and location of the tumor base on the retina begins with a digitized fundusphoto collage of the retina which must include the macula, the optic disc, and as much of the tumor as can be photographed [12]. The center of the optic disk and the posterior pole near the macula are readily identifiable landmarks on the collage. After these two locations are marked

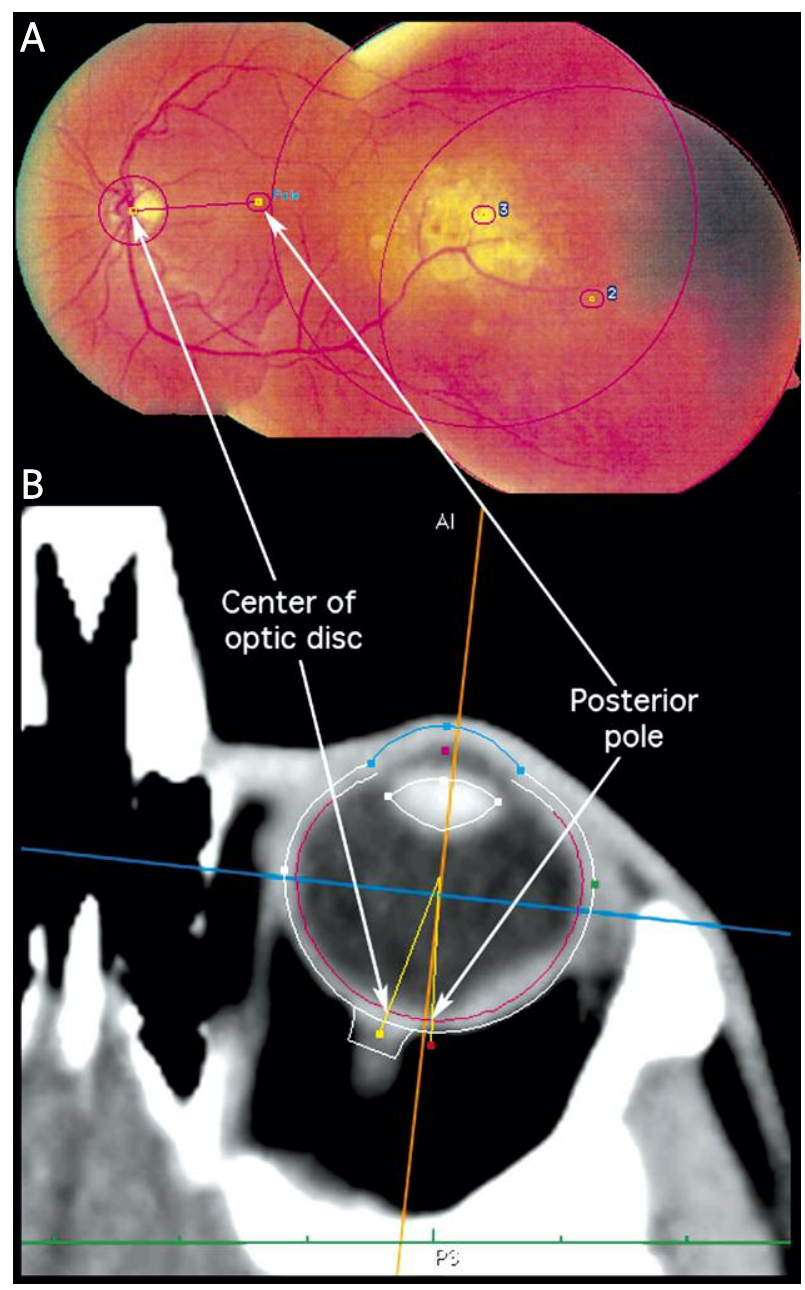

Fig. 2. A) A fundus photo collage assembled from 4 camera images. The posterior pole is close to the center of the leftmost image. The centers of photos 2 and 3 are marked. The $4^{\text {th }}$ image is mostly obscured by the other 3 so it has been ignored. The fundus collage can be calibrated and fused with 3D-CT imaging studies by finding the posterior pole and the center of the optic disc on the collage and in 3D-CT space. B) The size and shape of the eye, and the center of the optic disc are derived from a transverse axial CT slice that bisects the eye through the poles and the optic nerve

on the collage, as illustrated in Fig. 2A, the 3D coordinate of every pixel in the collage can be calculated relative to those landmarks once the 3D coordinates of those landmarks are determined. Returning the fundus collage to $3 \mathrm{D}$ space also requires that the radius of curvature of the retina be known, and, in what direction the camera was looking when photographing each of the smaller pictures that make up the collage. In Fig. 2A, the approximate centers of most of the photos that make up the collage are marked to indicate where the central ray of the camera was pointing when photographing that region of the retina.

The PS coordinates of the posterior pole, the center of the optic disc, and the radius of curvature of the retina initially default to the COMS recommended standard model of the eye. If available, a transverse axial CT image which bisects the eye through the anterior pole at the cornea, the lens, 
the posterior pole at the macula, and which shows the optic nerve entering the eye can be used to customize the PS model to match the spherical or oblate curvature of the actual patient eye as illustrated in Fig. 2B. In general, the slice spacing is too great, and the resolution of CT and MRI is too low to reliably determine tumor size and shape within the eye. However, by finding a common pair of landmarks in both the fundus photos and CT images, the pixels of the fundus collage can be transformed to 3D space and fused with the CT or MRI study to reveal the tumor location for a specific patient. Occasionally, the taller tumors are visible in CT and MRI and this has proven useful for confirming the fundus collage 3D transform. CT and MRI are also very useful companions to ultrasound for estimating the size, shape and location of anterior tumors which cannot be photographed.

The tumor location is determined by projecting the digitized fundus collage onto a two dimensional (2D) map of the retinal surface and then manually outlining the tumor base [12]. Using the earth as an analogy, the spherical surface of the earth is most commonly represented using the cylindrical Mercator projection in which size and shape distortion is minimal at the equator and maximum at the poles. In the eye, however, the region of greatest visual interest surrounds the posterior pole, not the equator. The retinal

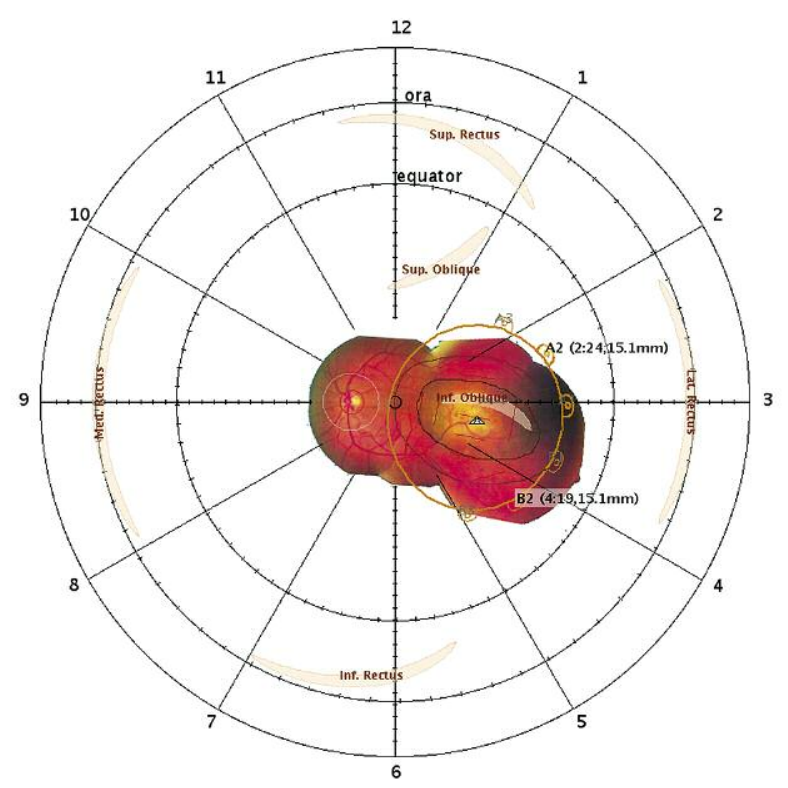

Fig. 3. Retinal diagram (RD) of a left eye, illustrating a $3.4 \mathrm{~mm}$ apical height, $10 \times 6 \mathrm{~mm}$ elliptically shaped tumor and a $2 \mathrm{~mm}$ retinal margin surrounding the base of the tumor. Also projected onto the diagram are generic approximations of where the six extraocular muscle insert into the underlying sclera, the shell of a COMS $14 \mathrm{~mm}$ plaque and its suture eyelets, the surgical suture coordinates of those eyelets expressed as meridian clock hour and chord distance from the limbus, and placeholder markers for the radionuclide seeds in the silicone carrier of the plaque. All objects, including the fundus-photo collage have been circumferentially warped as a function of latitude for correct mapping to the polar RD diagram (RD) is a polar map of the retinal surface from the posterior pole to the limbus. On a polar map, size and shape distortion is minimal at the polar center of the diagram and increases with the radial distance from the pole. For the RD illustrated in Fig. 3, observe that on the diagram the distance representing a unit distance (e.g. $1 \mathrm{~mm}$ ) in 3D space is radially constant, but stretches circumferentially with radial distance from the polar center of the diagram. For example, the RD circle representing the great circle of latitude at the equator of the eye is drawn with a smaller diameter, and the circumferential distance between $1 \mathrm{~mm}$ tic marks on the equatorial circle of the RD is smaller than for the circle on the RD which represents the circle of latitude at the ora serrata which lies in the anterior hemisphere of the eye. The circle of latitude at the ora is, of course, three dimensionally much smaller than the circle at the equator.

There is an exact transformation between the retinal surface in 3D space and the RD, and most objects of interest near the retina, such as the tumor apex, the extraocular muscle insertion locations, the plaque perimeter and the radiation sources can be projected onto the RD as illustrated in Fig. 3. Note that all 3D objects mapped onto the retinal diagram, including the fundus photo collage, must be circumferentially stretched according to their latitude as was illustrated for the circle of latitude at the ora.

After the perimeter of the tumor base has been outlined on the RD, the points defining the perimeter polygon can be transformed back into 3D coordinates on the retina, and a PTV-like margin added by expanding the perimeter polygon on the spherical and/or oblately shaped surface of the retina as determined from the CT images. A $2 \mathrm{~mm}$ margin is illustrated as a thin brown line surrounding the nominally elliptical tumor base in Fig. 3. The 3D model of the tumor is completed by using ultrasound imaging to determine the shape and height of the tumor dome resulting in an interactive 3D model that includes the ocular anatomy, the tumor, the plaque, the suture coordinates and the calculated radiation dosimetry as illustrated in Fig. 4.

Once the 3D model and its RD projection have been created, rival treatment planning options, such as the choice of radionuclide, and the number and location of radiation sources, may be quantitatively compared using a retina dosearea histogram. The dose-area histogram for the surface of the retina is calculated by first plotting a raster image analogous to the RD seen in Fig. 3, except that the image contains only the tumor and margin against a plain white background. The area on the spherical surface of the retina represented by a pixel on the rasterized RD varies with its latitude, so the area of each pixel in the RD image is calculated as a function of latitude, and then the coordinates of the center of that that pixel are transformed from RD space to 3D space and the dose delivered at that point is calculated. The areas are binned as a function of dose for the object they represent on the RD, and plotted in a manner analogous to the ubiquitous dose-volume histogram.

The objects for which dose-area will be calculated are: (1) the entire photosensitive surface of the retina represented by RD pixels that fall within the ora circle, (2) pixels that fall within the tumor perimeter anywhere on the diagram, (3) pixels that fall outside of the tumor perimeter and within 


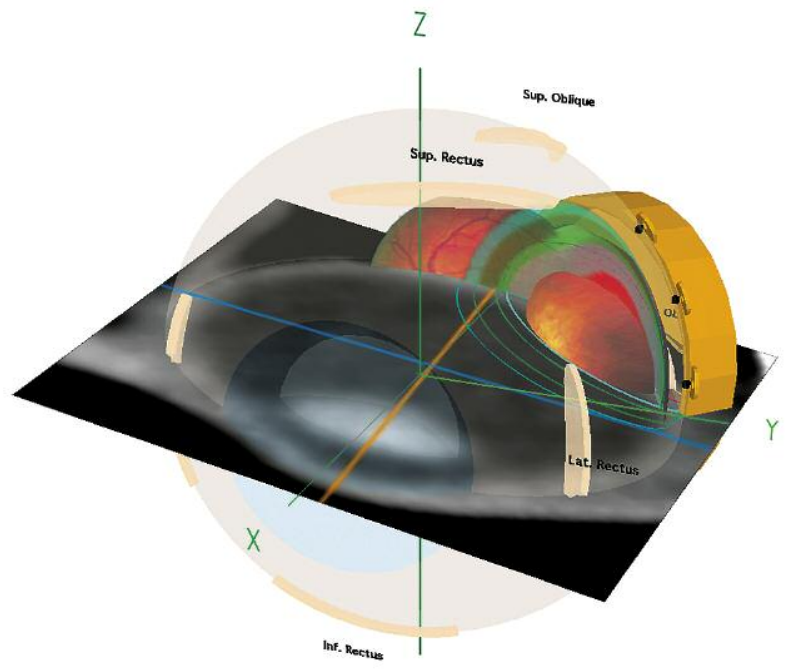

Fig. 4. 3D fusion of CT imaging, fundus photography, ultrasound measurements, plaque, suture eyelets, surgical coordinates and radiation dosimetry

the ora circle, (4) pixels that fall within the margin surrounding the tumor base, (5) pixels that fall outside of the margin and within the ora circle and (6) pixels that fall within the macular region which is approximated as a $5 \mathrm{~mm}$ diameter disc surrounding the posterior pole. In practice, this is easily accomplished by filling the tumor base and margin perimeter polygons on the raster image with different colors which encode what objects those pixels belong to and simply sorting according to the color of each pixel when binning the object areas. The raster image method is robust in that it works for polygons of arbitrary size and shape. The spatial resolution of the histogram will increase, as will the calculation time, as a function of the number of pixels that comprise the raster image.

\section{Results}

The surface area $A$ of a spherical dome, or equivalently, a spherical bowl is

$$
A=2 \pi r_{C} h
$$

where $r_{c}$ is the radius of curvature of the sphere and $h$ is the height of the dome, or equivalently, the depth of the bowl as illustrated in Fig. 5. The height $h$ can be calculated from the chord diameter $d$ of the dome at its base and the curvature of the sphere as

$$
h=r_{c}\left(1-\cos \left(\operatorname{asin}\left((d / 2) / r_{c}\right)\right)\right)
$$

For a $24 \mathrm{~mm}$ equatorial diameter spherical eye, and assuming that the retina is inset $1 \mathrm{~mm}$ from the external surface of the eye, the radius of curvature of the retina $r_{c}=11 \mathrm{~mm}$. The retinal surface area covered by a tumor with a circularly shaped base of chord diameter $d=10 \mathrm{~mm}$ is $83.08 \mathrm{~mm}^{2}$.

The spacial resolution of the histogram, and hence, the visual smoothness of the plotted dose-area curves, increases with the number of pixels that comprise the RD on the raster

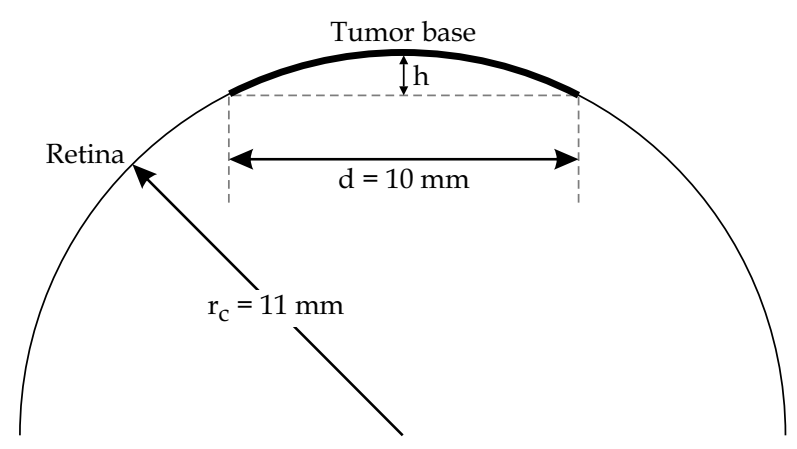

Fig. 5. Diagram illustrating the terms used in the analytical solution of the spherical surface of a tumor with a circular base of chord diameter $(d)$, spherical radius of curvature $\left(r_{c}\right)$ and dome height $(h)$

image. However, increasing the number of pixels proportionally increases the calculation time.

Table 1 summarizes results obtained when varying the RD image resolution for the same eye and tumor description for which the analytical solution was calculated above. The tumor is assumed to have a circular base of chord diameter $10 \mathrm{~mm}$ approximated by a polygon consisting of 36 edges and a retinal spherical radius of curvature of $11 \mathrm{~mm}$. Because the tumor perimeter is defined by a polygon, we expect the calculated area to be slightly less than the $83.08 \mathrm{~mm}^{2}$ derived from the analytical solution above. The third column in Table 1 is the number of colored

Table 1. The leftmost column is the diameter, measured in pixels, of the RD in the rasterized image that is used to calculate the RDAH. The retina was modeled as being spherical with a radius of curvature of $11 \mathrm{~mm}$. The second column is the number of polygon edges that were used to approximate the circular base of a $10 \mathrm{~mm}$ chord diameter tumor. The third column is the number of pixels that filled the polygon on the RD image. Note that although pixels on the RD image are all identical squares, the area that each pixel represents on the spherical surface varies with its radial distance from the pole of the RD. The rightmost column is the area on the spherical surface of the retina occupied by the tumor base. It is calculated by numerically integrating the areas of the pixels that filled the tumor polygon. The analytical solution for the area of this tumor on the spherical surface is $83.08 \mathrm{~mm}^{2}$

\begin{tabular}{lccc}
$\begin{array}{l}\text { RD diameter } \\
\text { (pixels) }\end{array}$ & Polygon edges & $\begin{array}{c}\text { Tumor base } \\
\text { (pixels) }\end{array}$ & $\begin{array}{c}\text { Calculated area } \\
\left(\mathrm{mm}^{2}\right)\end{array}$ \\
\hline 64 & 36 & 76 & 84.4 \\
\hline 128 & 36 & 292 & 81.4 \\
\hline 256 & 36 & 1180 & 82.3 \\
\hline 512 & 36 & 4752 & 82.9 \\
\hline 1024 & 36 & 18953 & 82.6 \\
\hline 2048 & 36 & 75811 & 82.7
\end{tabular}


Table 2. The description of Table 2 is the same as Table 1 except that here the number of polygon edges used to draw the tumor on the RD image is varied from 24 to 180 . The analytical solution for the area of this tumor on the spherical surface is $83.08 \mathrm{~mm}^{2}$. As expected, the numerical integration approaches the analytical solution as the perimeter of the polygon approaches a circle

\begin{tabular}{lccc}
$\begin{array}{l}\text { RD diameter } \\
\text { (pixels) }\end{array}$ & Polygon edges & $\begin{array}{c}\text { Tumor base } \\
\text { (pixels) }\end{array}$ & $\begin{array}{c}\text { Calculated area } \\
\left(\mathrm{mm}^{2}\right)\end{array}$ \\
\hline 1024 & 24 & 18796 & 82.0 \\
\hline 1024 & 36 & 18953 & 82.6 \\
\hline 1024 & 72 & 19022 & 82.9 \\
\hline 1024 & 180 & 19030 & 83.0
\end{tabular}

pixels that filled the polygon on the RD image. Recall that although pixels on the rasterized RD image are all identical squares, the area of spherical surface that each pixel represents varies with its radial distance, or latitude, from the pole of the RD. The rightmost column is the area on the spherical surface of the retina occupied by the tumor base. It is calculated by numerically integrating the areas of the pixels that filled the tumor polygon.

In Table 2 the number of polygon edges used to draw the tumor on the RD image is varied from 24 to 180 . As expected, the numerical integration approaches the analytical solution as the perimeter of the polygon approaches a circle. The dose-area histograms in the remainder of this work were calculated using a RD diameter of 1024 pixels which provides very smooth dose-area curves. Calculation speed was typically a few seconds on a 3.3 GHz Quad-Core Intel Xeon processor Apple MacPro computer. To illustrate how the RDAH might be used as a clinical tool, consider the example patient of Figs. 2 and 3. The tumor is in the left eye, it is $3.4 \mathrm{~mm}$ tall at the apex which was determined from ultrasound measurements, it has an elliptically shaped base of about $10 \times 6 \mathrm{~mm}$ which was digitized with a 147 edge polygon. The $2 \mathrm{~mm}$ retinal margin surrounding the base was automatically calculated by the PS software.

The $14 \mathrm{~mm}$ diameter COMS plaque is a good physical and dosimetric match to the $10 \mathrm{~mm}$ major axis of the tumor base. In Fig. 6, several rival treatment options are presented. In Fig. 6A the silicone seed carrier of the COMS $14 \mathrm{~mm}$ plaque has been fully loaded with 13 uniformly weighted I-125 seeds (model IAI-125A, IsoAid LLC, Port Richey, Florida, USA). In Fig. $6 \mathrm{~B}$ only the portion of the silicone seed carrier that conforms to the shape of the tumor base has been loaded with 7 uniformly weighted I-125 seeds. Fig. 6C demonstrates a similarly sized model CCA Ru-106 betaray plaque. Ru plaques are a viable alternative to $\mathrm{I}-125$ and Pd-103 plaques for tumors less than $4 \mathrm{~mm}$ tall. The Ru plaques are widely used in Europe $[2,3,18,19]$. The plaque in Fig. $6 \mathrm{D}$ is similar to $6 \mathrm{~B}$ except that the I- 125 seed closest to the macula has been removed and replaced by a pair of seeds in the two carrier positions adjacent to the position that was just vacated. These two seeds are weighted at half the strength of the other 6 seeds. Possible advantages of this spatially semi-conformal, non uniform redistribution of ra- dionuclide are that the two half-weighted seeds are further away from the macula than the seed they replaced, and they also happen to be oriented with their axes of maximum self-attenuation directed at the macula. In Fig. $6 \mathrm{E}$ we have a COMS $14 \mathrm{~mm}$ shell with a modified seed carrier [20] that has been semi-conformally loaded using the same seed pattern and radionuclide weighting distribution as Fig. 6D except that Pd-103 seeds [7] (model 200, Theragenics Corp., Buford, Georgia, USA) have been substituted for the I-125 seeds.

The plaque shells were all automatically centered below the geometric center, or centroid, of the tumor base and automatically rotated to balance the distance of the suture eyelets from the limbus in order to simplify the surgery. The seed carriers of the COMS plaques were manually rotated within the shell so that a roughly elliptically shaped subset of the seed positions in the carrier would spatially conform to the elliptical shape of the tumor base and retinal margin. In all cases the American Brachytherapy Society (ABS) recommended minimum dose of $85 \mathrm{~Gy}$ was prescribed to the apex of the tumor [21].

From left to right, the columns in Fig. 6 illustrate the radionuclide loading of the plaque, isodose lines on the retina, and lastly isodose lines on a meridian plane bisecting the eye through the tumor apex. The gray shaded hexagonal patches lining the concave surface of the Ru plaque model illustrate beta-ray patch-source modeling [13] of the relative distribution of the radionuclide embedded in the $\mathrm{Ru}$ plaque which enables the software to reproduce the central axis and surface dose-rate measurements provided by the manufacturer to within a few percent.

Retina dose-area histograms were calculated for the rival plans of Fig. 6. The results are presented in Fig. 7 wherein the curves plot the relative area as a function of dose to the objects of interest. Normal tissue tolerance for retina and the prescription dose of $85 \mathrm{~Gy}$ to the tumor apex are specifically marked along the top of the graphs. The heavy solid lines in all of these figures represent the Fig. 6B COMS $14 \mathrm{~mm}$ plaque conformally loaded with I-125 seeds. In Fig. 7A the dashed lines are the fully loaded COMS plaque (Fig. 6A), and the intermittent dash-dot lines are the Ru-106 beta-ray plaque (Fig. 6C). In Fig. 7B the dashed lines are the spatially semi-conformal COMS plaque (Fig. 6D) non-uniformly loaded with I-125 seeds. The intermittent dash-dot lines are the nearly equivalent modified COMS plaque [20] (Fig. 6E) non-uniformly loaded with Pd-103 seeds.

\section{Discussion}

The threshold dose for retinal damage is generally considered to be about 30-35 Gy. Emami et al. [22] report 5\%/5yr normal tissue tolerance for retina to be $45 \mathrm{~Gy}$ and $50 \% / 5 \mathrm{yr}$ tolerance to be $65 \mathrm{~Gy}$. Animal studies have shown damage to rods with $20 \mathrm{~Gy}$ and to cones with $100 \mathrm{~Gy}$. Although damage to photoreceptors has been reported, it is the inner retinal layers and retinal vascular endothelial cells that are the most affected, causing vessel closure and ischemic (capillary dropout) retinopathy which, in turn, leads to additional forms of damage including neovascularization which can induce secondary glaucoma, retinal detachment, and vitreous hemorrhage [9]. When the macula and fovea are 
A

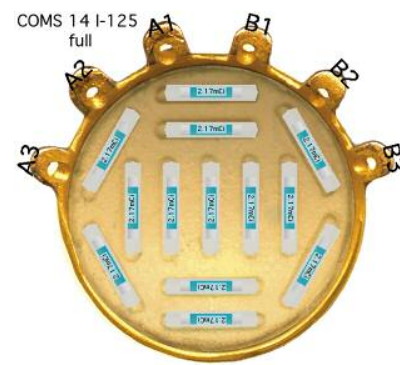

B
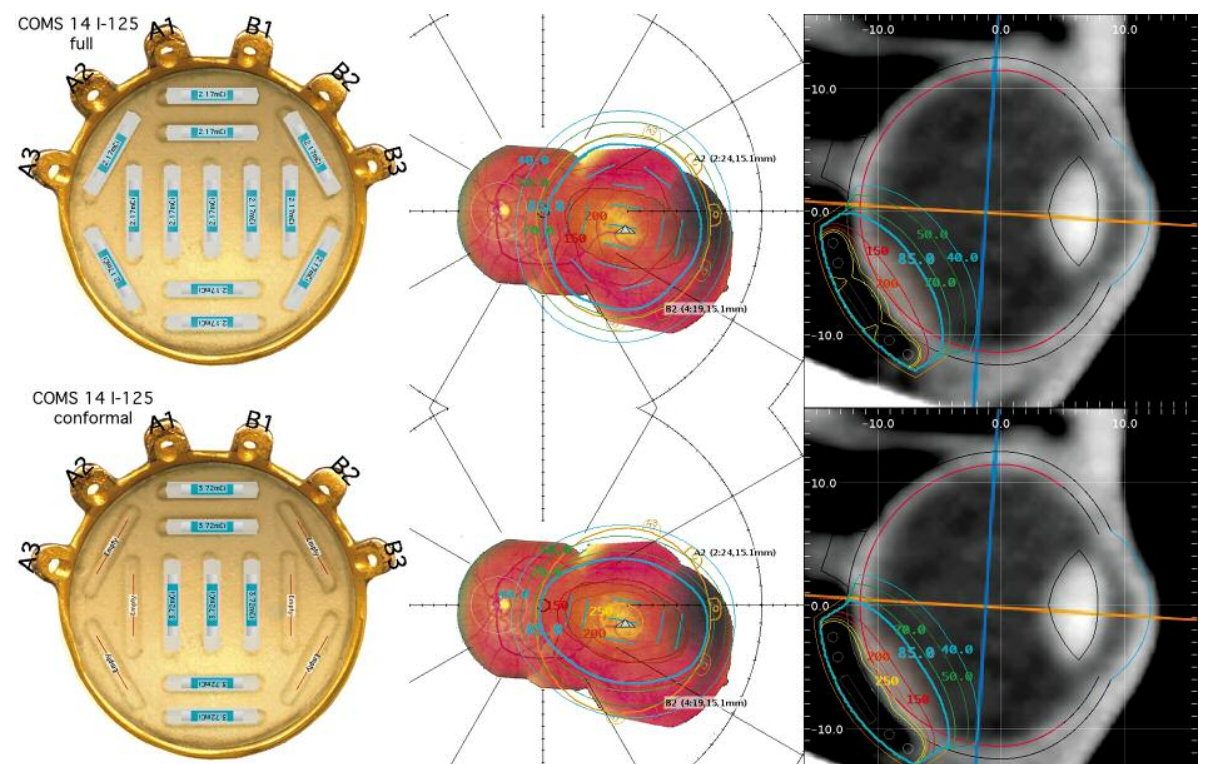

C

BEBIG CCA

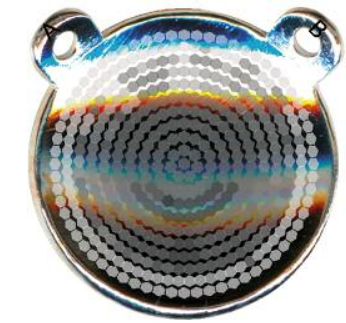

D

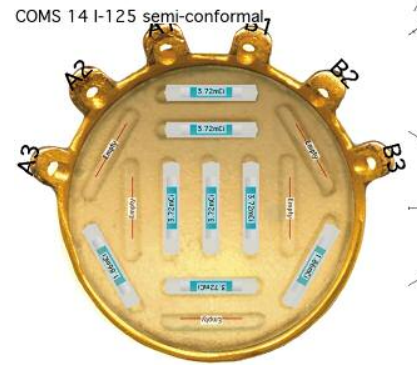

$\mathrm{E}$
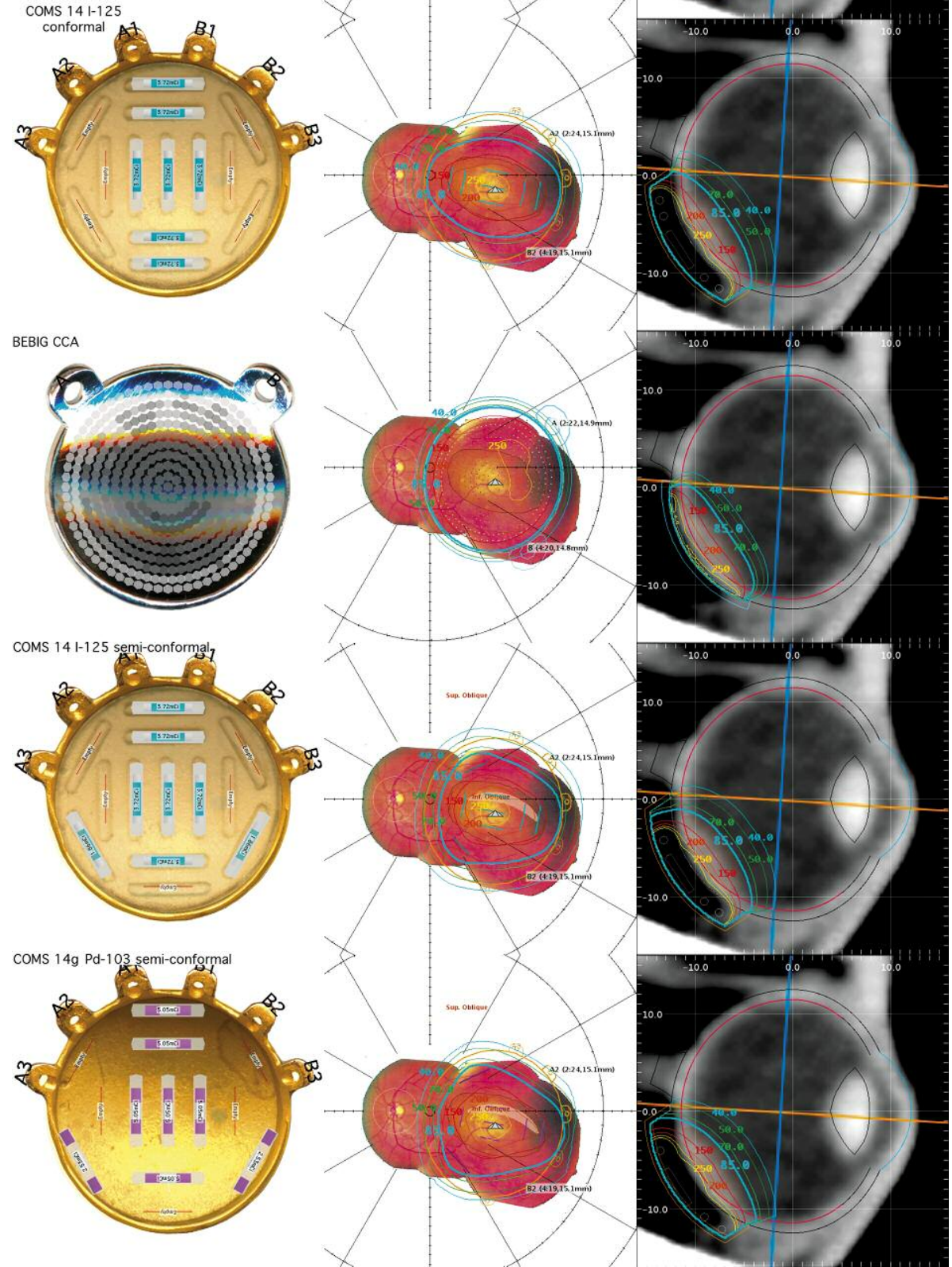

Fig. 6. Five rival treatment options for a $3.4 \mathrm{~mm}$ tall, elliptically shaped tumor. A) COMS $14 \mathrm{~mm}$ plaque fully loaded with I-125 seeds. B) COMS $14 \mathrm{~mm}$ plaque with its carrier rotated to enable a partial loading of the carrier with I-125 seeds to dosimetrically conform to the shape of the tumor and its $2 \mathrm{~mm}$ retinal margin. C) Ru-106 model CCA beta-ray plaque. D) Similar to B) except that the I-125 seed closest to the macula has been removed and replaced by two adjacent seeds of half weighting. E) COMS $14 \mathrm{~mm}$ shell modified with a gold insert in place of the silicone seed carrier and semi-conformally loaded as in D) except with Pd-103 seeds. In all cases the plaques were automatically centered under the tumor and a dose of 85 Gy was prescribed to the apex of the tumor. From left to right, the columns illustrate the radionuclide loading of the plaque, isodose lines on the retina, and isodose lines on a meridian plane bisecting the eye through the tumor apex. Isodose lines are plotted for the range 40 to 300 Gy. The yellow 300 Gy isodose line is unlabeled 

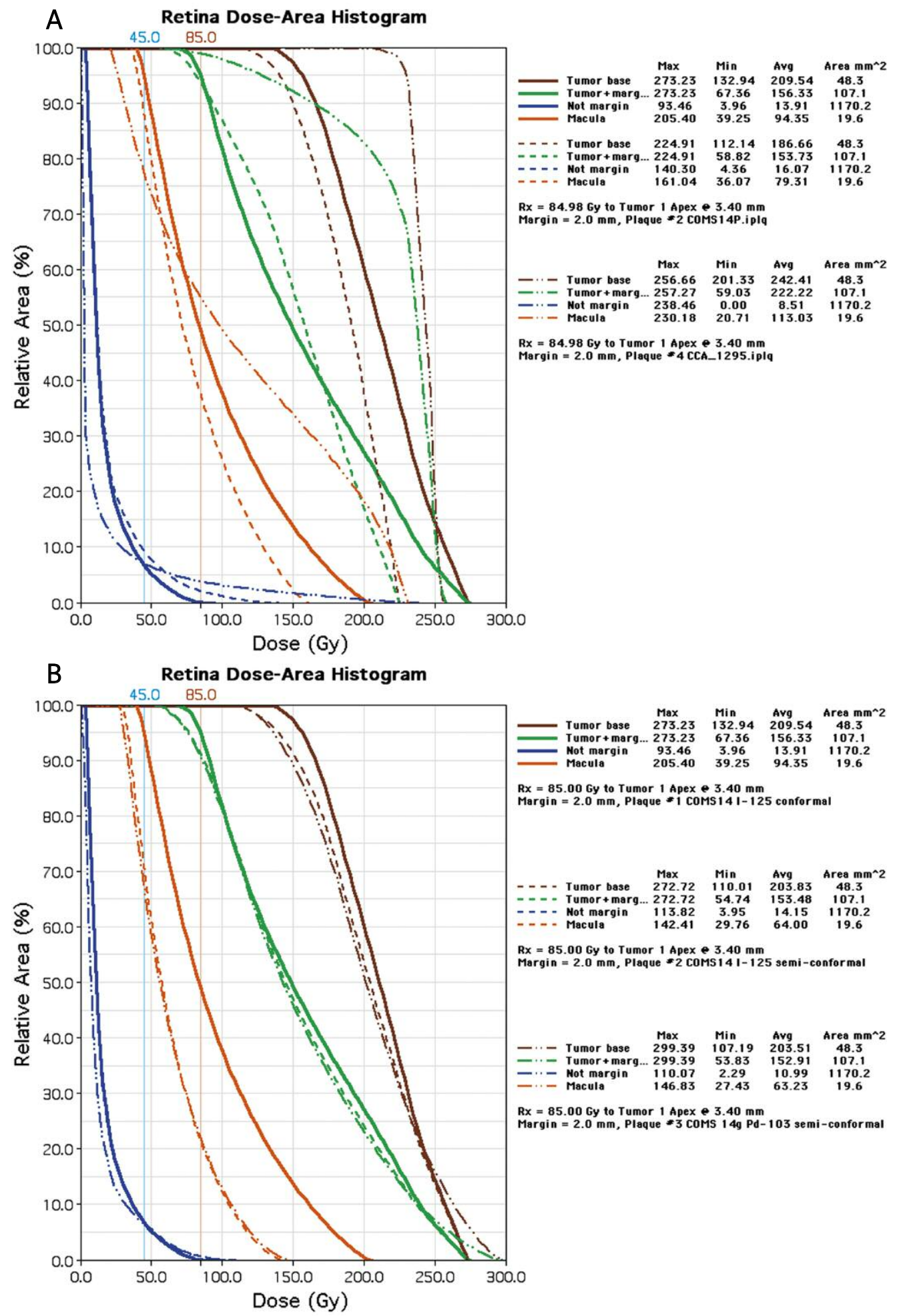

Fig. 7. A) Retina dose-area histograms for the rival plans presented in Figs. 6A, 6B and 6C. The dashed lines are the COMS $14 \mathrm{~mm}$ plaque (Fig. 6A) fully loaded with I-125 seeds. The heavy solid lines are the COMS $14 \mathrm{~mm}$ plaque (Fig. 6B) conformally loaded with I-125 seeds. The intermittent dash-dot lines are the model CCA Ru-106 beta-ray plaque (Fig. 6C). The blue curves are the areal dose to the photosensitive portion of the retina that lies outside the margin that surrounds the tumor base. The orange curves represent the macular region. These are the regions where the radiation dose should be minimized as best possible. B) Retina dose-area histograms for the plans presented in Figs. 6B, 6D and 6E. The heavy solid lines represent the COMS plaque conformally loaded with I-125 seeds (Fig. 6B). The dashed lines represent the semi-conformal COMS plaque (Fig. 6D) with the I-125 seed closest to the macula relocated to the adjacent carrier positions as two half-strength seeds. The intermittent dash-dot lines represent the gold-insert-modified COMS plaque (Fig. 6E) semi-conformally loaded with Pd-103 seeds 
involved, radiation maculopathy typically results in blindness $[8,9]$.

In Figs. 7A and $\mathrm{B}$, the orange and blue colored RDAH curves are of particular interest, because they plot relative area as a function of dose to the macular region and to the photosensitive portion of the retina that lies outside the PTVlike margin that surrounds the tumor base. These are the regions where one would like to reduce radiation dose with particular attention to areas which exceed normal tissue tolerance (e.g. 45 Gy).

With regard to the retina outside the margin (blue lines), the COMS plaque conformally loaded with I-125 sources (Fig. 6B), plotted as the thickest blue line, irradiates the least area to doses above $40 \mathrm{~Gy}$. The Ru beta-ray plaque irradiates the least area for doses below about $25 \mathrm{~Gy}$. With regard to the macula (orange curves), which happens to lie just above the posterior lip of the plaques in this particular example, the conformally loaded plaque (Fig. 6B), plotted as the thick orange lines, performs poorly. It delivers a much higher maximum dose to the macula and consistently irradiates a greater area of the macula as a function of dose. This occurs because it uses the fewest number of seeds of the highest individual activity and one of those seeds happens to be positioned very close to the macula.

In Figs. 6D and 7B observe that by removing the I-125 seed closest to the macula and redistributing its original activity between the two adjacent positions in the carrier, we get a spatially semi-conformal loading that significantly reduces the maximum dose in the macular region at the cost of only a small loss of coverage at the periphery of the margin.

In Figs. 6E and 7B we see that substituting Pd-103 (dashdot lines) for the I-125 (dashed lines) in Fig. 6D reduces the area receiving doses below $50 \mathrm{~Gy}$ at the cost of higher dose to about $15 \%$ of the tumor base and $5 \%$ of the margin. This is to be expected because the radial dose function is steeper for Pd-103 than for I-125 and the dose is prescribed to the apex of the tumor. In all of these rival plans, adequate dosimetric coverage of the tumor base and margin is achieved. The Ru beta-ray plaque (Fig. 6C) delivered good coverage of the tumor base and margin, and irradiated the least area at the low end of the dose range. Unfortunately, it also resulted in the highest macular dose. Arguably, it might be a good option for a circularly shaped tumor further away from the macula. In Fig. 7A we see that the fully loaded COMS plaque of Fig. 6A delivers a slightly higher maximum dose to the macula and irradiates a noticeably larger area of retina outside the margin (dashed blue lines in Fig. 7A) in the dose range above 25 Gy compared to its I- 125 rivals, the plaques of Figs. 6B and 6D.

Among rivals, the plan that delivers the prescribed dose to the tumor and margin while minimizing the area of macula (orange curves in Fig. 7) receiving doses greater than normal tissue tolerance (e.g. $45 \mathrm{~Gy}$ ) will likely be the plan of choice. Reducing the area of non-specific retina outside the margin (blue curves) receiving doses greater than normal tissue tolerance would be a secondary goal. Amongst the options presented in Fig. 6, the two plaques that are semiconformally loaded (Figs. 6D and 6E) deliver the least macula dose, as well as the least area outside the margin for dos- es above $40 \mathrm{~Gy}$, with the I-125 version irradiating slightly less area to doses greater than about $100 \mathrm{~Gy}$, and the Pd103 version slightly less area to doses below about $50 \mathrm{~Gy}$.

The radiation dose prescription recommendations [21] for episcleral plaque therapy which are currently in use are based on clinical experience gained at a time when there was great uncertainty regarding plaque dosimetry and surgical placement. Considering the advances in plaque design, treatment planning and delivery over the past decade, and the observation that vision-impairing complications appear to be a topic of great concern in the ophthalmology literature, it may be time to consider dose de-escalation studies.

\section{Conclusions}

The retina dose-area histogram provides a quantitative metric by which rival episcleral plaque treatment plan options may be quickly compared with regard to tumor and margin coverage, and possibly reducing the severity of subsequent radiation retinopathy and maculopathy. As with the dose-volume histogram, spatial location is lost in this method of analysis, so it must always be used as a companion to 2D and 3D isodose maps when making decisions regarding patient treatments.

\section{References}

1. Stallard HB. Radiotherapy for malignant melanoma of the choroid. Brit J Ophthalmol 1966; 50: 147-155.

2. Lommatzsch PK. Treatment of choroidal melanomas with Ru-106/Rh-106 beta ray applicators. Surv Ophthalmol 1974; 19: 85-100.

3. Lommatzsch PK. Irradiation of choroidal melanomas with Ru-106/Rh-106 applicators, 20 years' experience. Br J Ophthamol 1986; 70: 844-851.

4. Packer S, Rotman M. Radiotherapy of choroidal melanoma with iodine-125. Ophthalmol 1980; 87: 82-590.

5. Luxton G, Astrahan MA, Liggett PE et al. Dosimetric Calculations and Measurements of Gold Plaque Ophthalmic Irradiators using Iridium-192 and Iodine-125 Seeds. Int J Radiat Oncol Biol Phys 1988; 15: 167-176.

6. Earle J, Kline RW, Robertson DM. Selection of iodine 125 for the collaborative ocular melanoma study. Arch Ophthalmol 1987; 105: 763-764.

7. Finger PT, Berson A, Ng T et al. Palladium-103 plaque radiotherapy for choroidal melanoma: an 11-year study. Int J Radiat Oncol Biol Phys 2002; 54: 1438-1445.

8. Finger PT, Chin KJ, Yu GP. Risk factors for radiation maculopathy after ophthalmic plaque radiation for choroidal melanoma. Am J Ophthalmol 2012; 149: 608-615.

9. Finger PT. Radiation retinopathy is treatable with anti-vascular endothelial growth factor bevacizumab (avastin). Int J Radiat Oncol Biol Phys 2008; 70: 974-977.

10. Astrahan MA, Luxton G, Jozsef G et al. An interactive treatment planning system for ophthalmic plaque radiotherapy. Int J Radiat Oncol Biol Phys 1990; 18: 679-687.

11. Astrahan MA, Luxton G, Jozsef G et al. Optimization of I-125 ophthalmic plaque brachytherapy. Med Phys 1990; 17: 1053-1057.

12. Evans MDC, Astrahan MA, Bate R. Tumor localization using fundus view photography for episcleral plaque therapy. Med Phys 1993; 20: 769-775.

13. Astrahan MA. A patch source model for treatment planning of ruthenium ophthalmic applicators. Med Phys 2004; 30: 1219-1228. 
14. Astrahan MA. Improved treatment planning for COMS eye plaques. Int J Radiat Oncol Biol Phys 2005; 61: 1227-1242.

15. Astrahan MA, Luxton G, Pu Q et al. Conformal episcleral plaque therapy. Int J Radiat Oncol Biol Phys 1997; 39: 505-519.

16. Astrahan MA. Preplanning of Suture Coordinates for Episcleral Plaque Therapy. Proc. 51 $1^{\text {st }}$ Annual Meeting of the Amer. Assoc. Physicists in Medicine. Med Phys 2009; 36: 2536.

17. Drzymala RE, Mohan R, Brewster L et al. Dose-Volume Histograms. Int J Rad Oncol Biol Phys 1991; 21: 71-78.

18. Tjho-Heslinga RE, Davelaar J, Kemme HM et al. Results of ruthenium irradiation of uveal melanomas: the Dutch experience. Radiother Oncol 1999; 53: 133-137.

19. Seregard S, aft Trampe E, Lax I et al. Results following episcleral ruthenium plaque radiotherapy for posterior uveal melanoma. The Swedish experience. Acta Ophthalmol Scand 1997; 75: 11-16.

20. Astrahan MA, Szechter A, Finger P. Design and dosimetric considerations of a modified COMS plaque: the reusable seedguide insert. Med Phys 2005; 32: 2706-2716.

21. Nag S, Quivey JM, Earle JD et al. The American Brachytherapy Society Recommendations for Brachytherapy of Uveal Melanomas. Int J Radiat Oncol Biol Phys 2003; 56: 544-555.

22. Emami B, Lyman J, Brown A et al. Tolerance of normal tissue to therapeutic irradiation. Int J Radiat Oncol Biol Phys 1991; 21: 109-122. 